\title{
Modeling and simulation of weld solidification cracking part II
}

\section{A model for estimation of grain boundary liquid pressure in a columnar dendritic microstructure}

\author{
J. Draxler $^{1} \cdot$ J. Edberg ${ }^{1} \cdot$ J. Andersson ${ }^{2} \cdot$ L.-E. Lindgren ${ }^{1}$
}

Received: 23 October 2018 / Accepted: 27 May 2019 / Published online: 21 June 2019

(C) The Author(s) 2019

\begin{abstract}
Several advanced alloy systems are susceptible to weld solidification cracking. One example is nickel-based superalloys, which are commonly used in critical applications such as aerospace engines and nuclear power plants. Weld solidification cracking is often expensive to repair, and if not repaired, can lead to catastrophic failure. This study, presented in three papers, presents an approach for simulating weld solidification cracking applicable to large-scale components. The results from finite element simulation of welding are post-processed and combined with models of metallurgy, as well as the behavior of the liquid film between the grain boundaries, in order to estimate the risk of crack initiation. The first paper in this study describes the crack criterion for crack initiation in a grain boundary liquid film. The second paper describes the model for computing the pressure and the thickness of the grain boundary liquid film, which are required to evaluate the crack criterion in paper 1. The third and final paper describes the application of the model to Varestraint tests of Alloy 718. The derived model can fairly well predict crack locations, crack orientations, and crack widths for the Varestraint tests. The importance of liquid permeability and strain localization for the predicted crack susceptibility in Varestraint tests is shown.
\end{abstract}

Keywords Solidification cracking $\cdot$ Hot cracking $\cdot$ Varestraint testing $\cdot$ Computational welding mechanics $\cdot$ Alloy 718

\section{Introduction}

In the first paper of this study, a weld solidification crack (WSC) criterion was developed [1]. To evaluate the criterion in a given grain boundary liquid film (GBLF), the liquid pressure and thickness of the film must be known. The current paper describes a model for estimating these quantities, which is inspired by the RDG model proposed by Rappaz et al. [2]. The RDG model estimates the interdendritic liquid pressure drop to cavitation in

Recommended for publication by Study Group 212 - The Physics of Welding

Electronic supplementary material The online version of this article (https://doi.org/10.1007/s40194-019-00761-w) contains supplementary material, which is available to authorized users.

$\triangle$ J. Draxler

joar.draxler@1tu.se

1 Luleå University of Technology, 97187 Luleå, Sweden

2 University West, 46132 Trollhättan, Sweden a columnar dendritic microstructure. Suyitno et al. [3] compared eight hot cracking criteria in the simulation of DC casting of aluminum alloys. They found that the RDG model best reproduced the experimental trends. However, this model is limited by some shortcomings. One of them is that localization of strains at grain boundaries is not considered [4]. This shortcoming was addressed by Coniglio et al. [5]. Instead of assuming that strain is localized evenly between dendrites as in the RDG model, they assumed it to be localized evenly between grains.

The GBLF pressure model presented in the current paper is inspired by the RDG model and its improvements by Coniglio. In the proposed model, the pressure of the liquid is computed by a combination of Poiseuille parallel-plate flow and Darcy porous flow. Poiseuille flow is used in regions with less than 0.1 fractions of liquid, while Darcy flow is used in regions with more than 0.1 fractions of liquid. The permeability developed by Heinrich et al. [6] was used for the Darcy flow computations. It is considered more accurate than the Carman-Kozeny permeability [6], which is commonly used in the RDG model.

In the proposed model, a temperature-dependent length scale is used to account for strain localization in GBLFs. 
Instead of assuming that strain is always evenly partitioned between grains, as suggested by Coniglio, the degree of partitioning is assumed to vary during the solidification.

The model was evaluated on Varestraint tests of alloy 718 , and the evolutions of GBLF permeability, pressure, and thickness were studied.

\section{Model development}

The development of the model used for computing the pressure and thickness of a given GBLF is presented below. First, the method for computing GBLF orientation is presented, and then, the solidification model for the liquid in the GBLF is introduced. After that, a model for computing GBLF thickness from the macroscopic mechanical strain field of an FE model is presented. Finally, it is shown how a combination of Darcy's law and Poiseuille parallel-plate flow can be used to compute the pressure within a GBLF.

\subsection{GBLF orientation}

To compute the pressure in a given GBLF, the orientation of the GBLF must be known. The GBLF orientation in the fusion zone, FZ, of a weld depends on the solidification process. Normally, when the base and weld metal have the same crystal structure, the molten metal in the FZ starts to solidify from the fusion boundary with a cellular solidification mode [7]. At a short distance from the fusion boundary, if the welding speed is not too high, the solidification mode shifts to a columnar dendritic mode due to the increase in constitutional supercooling, which results in columnar grains. If the welding speed is high enough, the constitutional supercooling can continue to increase and the solidification mode can again change and go from columnar dendritic to equiaxed dendritic. However, in this study, we are interested in TIG welding at low welding speeds. The degree of constitutional supercooling is therefore assumed to never be large enough so that a transition from columnar to equiaxed dendritic solidification can occur.

Alloys with fcc or bec structure grow in the $\langle 100\rangle$ directions during solidification. They strive to grow in the orientation of the $\langle 100\rangle$ direction that is closest aligned with the temperature gradient of the liquidus isotherm [7]. Because the temperature gradient at the grain tip changes direction when the grains grow, grains may shift their growth orientation during the solidification in order to grow in the most favorable $\langle 100\rangle$ direction. Columnar grains can adjust their growth orientation either by bowing or by renucleation [7]. If we assume that the change in growth direction only occurs by bowing, and that there is no undercooling to solidification, the grain growth will always be normal to the liquidus isotherm, which results in curved columnar grains, extending all the way from the fusion boundary to the weld centerline. The rate of growth is then the same as the velocity of the liquidus isotherm in the direction of the temperature gradient. The above assumptions enable us to compute the grain axis solely from the temperature field of the weld.

With this grain axis, we associate a corresponding GBLF by assuming that the film extends along a curve that coincides with the grain axis, offset by the radius of the grain. We call this curve the GBLF axis. Furthermore, we define the normal direction of a GBLF to be in the same direction as the maximum macroscopic strain rate perpendicular to the GBLF axis. The computation of the normal direction from the macroscopic strain field is discussed in Section 2.4. The GBLF axis of a given GBLF is computed from the weld temperature field as follows. Consider a tip of a grain whose growth direction is normal to the liquidus isotherm with zero undercooling to solidification. Let $\mathbf{G}_{L}$ be the temperature gradient and $\mathbf{R}_{L}$ be the solidification velocity of the grain tip, with magnitudes $G_{L}$ and $R_{L}$, respectively. At the grain tip, the material derivative of the temperature field, $T$, is zero

$$
\frac{D T}{D t}=\frac{\partial T}{\partial t}+\mathbf{G}_{L} \cdot \mathbf{R}_{L}=0
$$

Given that $\mathbf{R}_{L}$ is in the same direction as $\mathbf{G}_{L}$ (because the growth direction is normal to the liquidus isotherm), $R_{L}$ can be solved for from Eq. 1, and $\mathbf{R}_{L}$ can then be expressed as

$\mathbf{R}_{L}=-\frac{1}{G_{L}^{2}} \frac{\partial T}{\partial t} \mathbf{G}_{L}$

Let $\mathbf{r}(t)$ be the location of the grain tip. The vector $\mathbf{r}$ will then trace out the grain axis, it can be determined by

$\frac{d \mathbf{r}}{d t}=\mathbf{R}_{L}$

where $\mathbf{R}_{L}$ is given by Eq. 2. Now, consider a GBLF associated with a grain axis $\mathbf{r}$, which is obtained by integrating (3). To integrate $\mathbf{r}$ in Eq. 3, we provide an initial condition $\mathbf{r}\left(t_{0}\right)=\mathbf{r}_{0}$, where $\mathbf{r}_{0}$ is a given point on the GBLF axis. $t_{0}$ is the time when the liquidus isotherm passes the point $\mathbf{r}_{0}$. Equation 3 is integrated from the temperature field obtained from a computational welding mechanics model, which is decribed in part III of this study [8]. This is done using a fourth-order Runge-Kutta method. By integrating forward in time from $t_{0}$, the part of the GBLF axis that aligns with the weld centerline can be obtained. The integration continues until the weld heat input is terminated. Further, by integrating backward in time from $t_{0}$, the second part 
Fig. 1 Schematic showing the procedure of integrating the axis of a GBLF that crosses the point $\mathbf{r}_{0}$

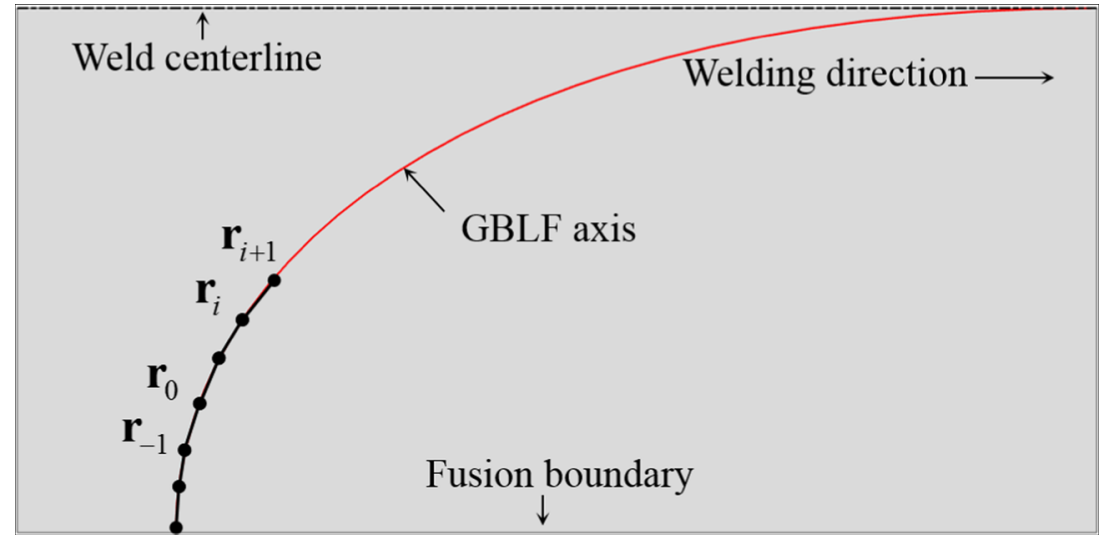

of the GBLF axis that intersect with the fusion boundary can be constructed. Here, the integration is stopped when the calculated value of $\mathbf{r}(t)$ at a time increment is more than $5{ }^{\circ} \mathrm{C}$ below the liquidus temperature, which ensures that the GBLF axis ends at the fusion boundary. Figure 1 illustrates the integration process.

\subsection{Undercooling}

In the above growth model, the undercooling at the dendritic tip was neglected. However, in rapid solidification processes such as welding, the undercooling can be substantial. In order to justify our assumption of neglected undercooling, we have used a model by Foster et al. [9] to compute the undercooling as a function of the solidification velocity for alloy 718 as follows.

The total undercooling at a dendritic tip can be expressed as the sum of four contributions [10]:

$$
\Delta T=\Delta T_{C}+\Delta T_{R}+\Delta T_{T}+\Delta T_{K}
$$

where $\Delta T_{C}, \Delta T_{R}, \Delta T_{T}$, and $\Delta T_{K}$ are the constitutional, curvature, thermal, and kinetic undercoolings, respectively. In welding, $\Delta T_{C}$ and $\Delta T_{R}$ are normally the dominating contributions to the total undercooling. Kurz et al. [11] have developed the KGT model to compute $\Delta T_{C}$ for binary alloys at both low and high solidification velocities. Rappaz et al. [12] extend the KGT model to multicomponent alloys and used it to study the dendrite growth in electron beam welding of a Fe-Ni-Cr alloy. Foster et al. [9] used the extended KGT model to compute the undercooling in laser welding of alloy 718. This model with data from Foster et al. [9] was used to calculate the undercooling for alloy 718 for solidification velocities in the range $10^{-2} \leq R_{L} \leq 10^{3}$ $\mathrm{mms}^{-1}$. The model depends on the temperature gradient, which was assumed to vary linearly between $8 \times 10^{4}$ and $10^{8}{ }^{\circ} \mathrm{Cm}^{-1}$, when $R_{L}$ goes from 1 to $1000 \mathrm{mms}^{-1}$. When $R_{L}<1 \mathrm{mms}^{-1}, G_{L}$ was assume to have the constant value $8 \times 10^{4}{ }^{\circ} \mathrm{Cm}^{-1}$. The value $8 \times 10^{4}{ }^{\circ} \mathrm{Cm}^{-1}$ was obtained from a computational welding mechanics model of a Varestraint test of alloy 718 with a welding speed of $1 \mathrm{mms}^{-1}$, see
Fig. 2 Calculated undercooling as a function of solidification velocity for alloy 718

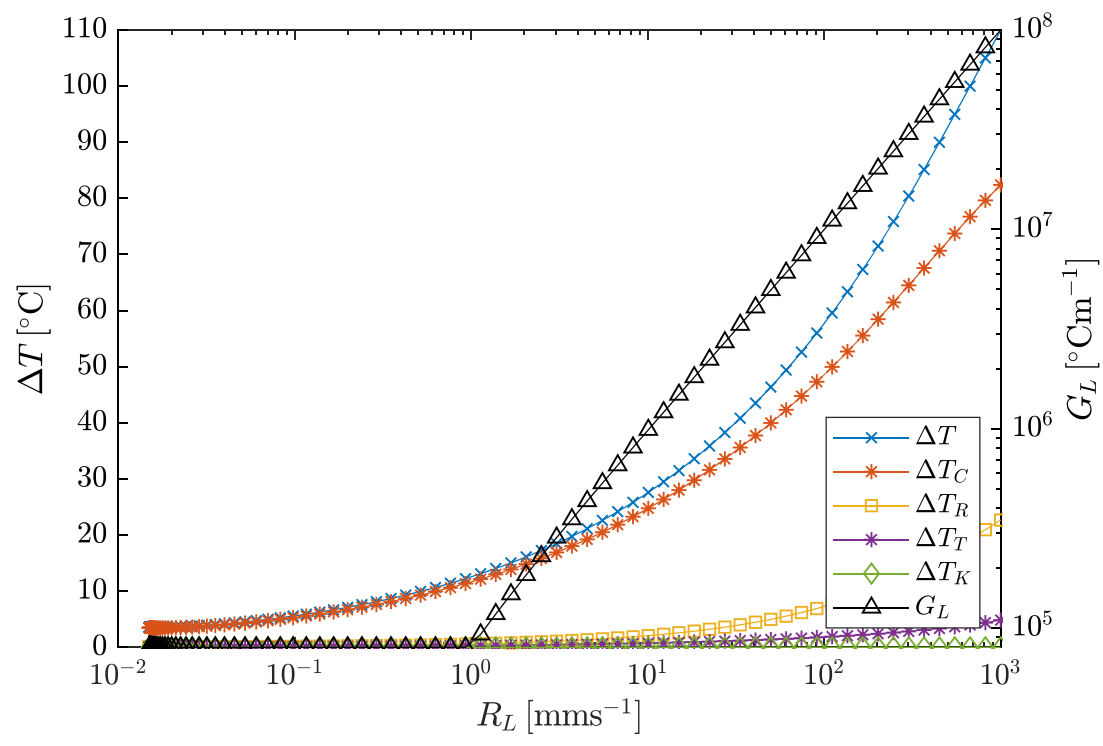


part III of this work [8]. Figure 2 shows the calculated total undercooling and its different contributions. As can be seen from the Figure, the largest contributions come from $\Delta T_{C}$ and $\Delta T_{R}$, while the contributions from $\Delta T_{T}$ and $\Delta T_{K}$ are negligible. More details on the models that were used to construct this Figure can be found in the Appendix.

In this work, we are interested in TIG welding with a welding speed of $1 \mathrm{mms}^{-1}$ in alloy 718 . At that low welding speed, $\Delta T$ is equal to $12.5^{\circ} \mathrm{C}$, as can be seen from Fig. 2 . That is less than $1 \%$ of $T_{l}$ for alloy 718 , and it will only shift the liquidus isotherm approximately $0.15 \mathrm{~mm}$ when $G_{L}=8 \times 10^{4}{ }^{\circ} \mathrm{Cm}^{-1}$. We therefore neglect the effect of the undercooling for this low welding speed.

\subsection{GBLF solidification model}

The solidification of the GBLF is an important part of computing the GBLF pressure. It determines the solidification temperature interval, which in turn determines the length of the GBLFs. It also determines the rate of solidification, and therefore, the rate of solidification shrinkage.

The solidification of multicomponent alloys is complex to model. To simplify the solidification of the GBLF, we assumed that it is governed by a multicomponent Scheil-Gulliver model [13]. A significant advantage of the Scheil-Gulliver model is its simplicity. The fraction of solid vs. temperature curve can easily be determined by a thermodynamic software such as Thermo-Calc [13]. However, the Scheil-Gulliver model has the following limitations: back diffusion from the liquid phase to the solid phase is neglected, diffusion in the liquid phase is assumed to be infinity fast, and the solidification front is assumed to be planar.

For the first limitation, the cooling rates in welding are often very high, which gives less time for back diffusion to occur. Thus, a considerable amount of back diffusion may only occur for high-diffusion elements such as carbon. For the second limitation, there are always convective currents in the weld pool that result in low-concentration gradients. Thus, at temperatures above the liquidus temperature, the assumption of complete diffusion in the liquid phase is valid. However, at lower temperatures, the permeability is low, and therefore, the convective currents in the liquid may be small. Thus, in this case, the assumption is less valid. The third limitation of a planar solidification front is not valid when we have a dendritic solidification mode, which imposes a curved solidification front. The curved solidification front leads to an undercooling to solidification. However, as was seen in Section 2.2, this undercooling is small for the low welding speeds that we are interested in in this work.

To estimate GBLF solidification using the ScheilGulliver model, the dendritic solid-liquid interfaces of the GBLF are approximated as planar, as shown in Fig. 3.

Let $2 h_{0}$ be the undeformed thickness of the flat GBLF. The undeformed thickness is defined as the GBLF thickness that results when no thermal or mechanical strains act on the GBLF. By assuming that the two opposing dendritic interfaces of the undeformed GBLF are separated by the primary dendrite arm spacing $\lambda_{1}, h_{0}$ can be written as (see Fig. 3)

$h_{0}=\frac{\lambda_{1}}{2}\left(1-f_{s}\right)$

where $f_{s}$ is the fraction of solid given by the ScheilGulliver model. This corresponds to a grain boundary with a low misorientation angle, which was chosen due to its simplicity. A grain boundary with a large misorientation angle is more messy and a larger value than $\lambda_{1}$ should be used in Eq. 5. The deformed GBLF thickness is derived later in Section 2.4.

The primary dendrite arm spacing is related to the solidification process, and in this study, it is estimated from the following expression [4]

$\lambda_{1}=\frac{C_{1}}{\left(G_{L}\right)^{1 / 2}\left(R_{L}\right)^{1 / 4}}$

where $C_{1}$ is a parameter. The $R_{L}$ term can be replaced with the cooling rate by substituting Eq. 2 into Eq. 6, which gives

$\lambda_{1}=\frac{C_{1}\left(G_{L}\right)^{1 / 4}}{\left(-\frac{\partial T}{\partial t}\right)^{1 / 4}}$

All terms in Eq. 7 are evaluated at the intersection between the GBLF axis and the liquidus isotherm. The $C_{1}$ parameter is determined by inverse modeling such that the computed $\lambda_{1}$ value agrees with the measured $\lambda_{1}$ value from an experiment at a given location [8].
Fig. 3 a Schematic of a GBLF. b GBLF approximated with planar interfaces

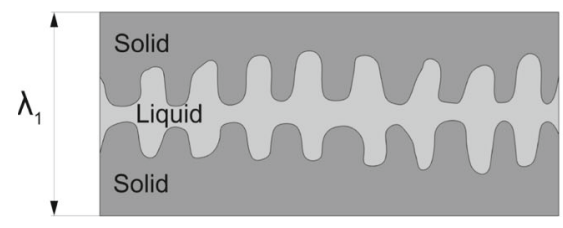

(a)

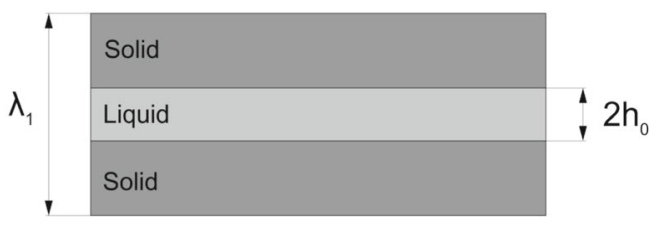

(b) 
The solidification speed $v^{*}$ of the solid-liquid interface of the idealized GBLF shown in Fig. $3 \mathrm{~b}$ can now be computed as the negative time derivative of $h_{0}$ in Eq. 5, which gives

$v^{*}=\frac{\lambda_{1}}{2} \frac{d f_{s}}{d T} \frac{d T}{d t}$

In this study, we assume that all liquid that remains when the temperature drops to solidus (which is given by the Scheil-Gulliver model) will instantly solidify. Thus, if the liquid can flow to the $T_{s}$ isotherm due to, e.g., tensile deformation of the GBLF, it will instantly solidify when it reaches this isotherm.

All temperature-dependent variables above are evaluated from the macroscopic temperature field obtained from an FE model of the welding process; see part III of this study [8] for more details.

\subsection{GBLF thickness}

In this section, we show how the deformed GBLF thickness, $2 h$, can be estimated from the macroscopic mechanical strain field of a finite element computational welding mechanics model.

\subsubsection{GBLF thickness derivation}

During solidification of the weld metal, deformation can strongly localize in the weak GBLFs. To compute the deformed GBLF thickness, we consider an arbitrary location on the axis of a given GBLF. At this location, we assume that all macroscopic mechanical strains, normal to the GBLF axis and within a distance $2 h+l_{0}$, will localize in the GBLF during the infinitesimal time $d t$, as shown in Fig. 4. Here, $l_{0}$ is a length scale that represents the amount of surrounding solid phase of the GBLF that can transmit normal tensile loads. The value of $l_{0}$ depends on the ability of the solid phase to transmit loads, and therefore, changes during the solidification of the alloy. This is further discussed in Section 2.4.3. In the above assumption, we have assumed that the solid phase is much stiffer than the liquid phase such that all mechanical strains are localized in the GBLF. We can now estimate $h$ as follows. Let $\varepsilon^{m}$ be the macroscopic mechanical strain tensor obtained from a computational welding mechanics model. With the above reasoning, the velocity of the solid-liquid interface of the GBLF can be written as (see Fig. 4)

$\dot{h}=\left(h+\frac{l_{0}}{2}\right) \dot{\varepsilon}_{\perp, \max }^{m}-v^{*}$

where $v^{*}$ is given by Eq. $8 . \dot{\varepsilon}_{\perp, \max }^{m}$ in Eq. 9 is the largest macroscopic mechanical strain rate in a plane normal to the GBLF axis of the GBLF, evaluated on the GBLF axis, which is further discussed in Section 2.4.2. Equation 9 can be integrated with a Euler backward method, which gives

${ }^{i+1} h= \begin{cases}\frac{2^{i} h+\Delta t\left({ }^{i+1} l_{0}{ }^{i+1} \dot{\varepsilon}_{\perp, \text { max }}^{m}-2^{i+1} v^{*}\right)}{2\left(1-\Delta t^{i+1} \dot{\varepsilon}_{\perp, \text { max }}^{m}\right)}, & { }^{i+1} h>h_{\text {min }} \\ h_{\text {min }}, & i+1 h \leq h_{\text {min }}\end{cases}$

where $i$ is the index of the time increment and $\Delta t$ is the time step. $h_{\min }$ is a cut-off value which ensures that division by zero is avoided when we later solve for the liquid pressure. A value of $0.01 \mu \mathrm{m}$ was used for $h_{\min }$ in this study.

\subsubsection{Maximum normal strain rate to the GBLF axis}

$\dot{\varepsilon}_{\perp, \text { max }}^{m}$ in Eq. 9 is computed as follows. We assume that the normal to the GBLF at a given location is always oriented parallel to the direction of $\dot{\varepsilon}_{\perp, \max }^{m}$. In this way, the normal deformation of the GBLF is maximized, which is assumed to be most detrimental. The mechanical strain rate tensor is determined with the central difference

${ }^{i} \dot{\varepsilon}^{m}=\frac{{ }^{i+1} \varepsilon^{m}-{ }^{i-1} \varepsilon^{m}}{2 \Delta t}$

Let $x y z$ be the global Cartesian coordinate system of the computational welding mechanics model that is used to determined $\varepsilon^{m}$. Further, let $x^{\prime} y^{\prime} z^{\prime}$ be a local Cartesian coordinate system whose $z^{\prime}$ axis is parallel to the tangent of the GBLF axis and with origin on the GBLF axis where we want to evaluate $\dot{\varepsilon}^{\prime m}$. The components of the $\dot{\varepsilon}^{m}$ tensor in the $x^{\prime} y^{\prime} z^{\prime}$ system are obtained from

$\left[\dot{\varepsilon}^{m}\right]^{\prime}=[\mathbf{Q}]\left[\dot{\varepsilon}^{m}\right][\mathbf{Q}]^{T}$

where $\mathbf{Q}$ is the transformation tensor from the $x y z$ system to the $x^{\prime} y^{\prime} z^{\prime}$ system. Because $z^{\prime}$ is tangent to the GBLF axis, $\dot{\varepsilon}_{\perp, \text { max }}^{m}$ is given as the largest eigenvalue of the matrix $\left[\dot{\varepsilon}^{m}\right]_{2 x 2}^{\prime}$, where $\left[\dot{\varepsilon}^{m}\right]_{2 x 2}^{\prime}$ is the $2 \times 2$ submatrix of $\left[\dot{\varepsilon}^{m}\right]^{\prime}$ that contains the $11,12,21$, and 22 components of the matrix $\left[\dot{\varepsilon}^{m}\right]^{\prime}$.

\subsubsection{Strain partition length}

The strain partition length $l_{0}$ in Section 2.4.1 depends on several features of the solidifying weld metal. For example, it is affected by the degree of coalescence and interlocking of dendrites and grains that surrounds the GBLF, and also by the GBLF morphology. In this study, we estimate $l_{0}$ from the temperature field and primary dendrite arm spacing as follows. At the liquidus temperature, the GBLF in the FZ just starts to form. Therefore, no strain localization can occur, which gives $l_{0}=0$. At the coherent temperature, the dendrites of individual grains start to coalescence such that the solidifying structure can transmit small tensile 
Fig. 4 Strain partitioning in a GBLF

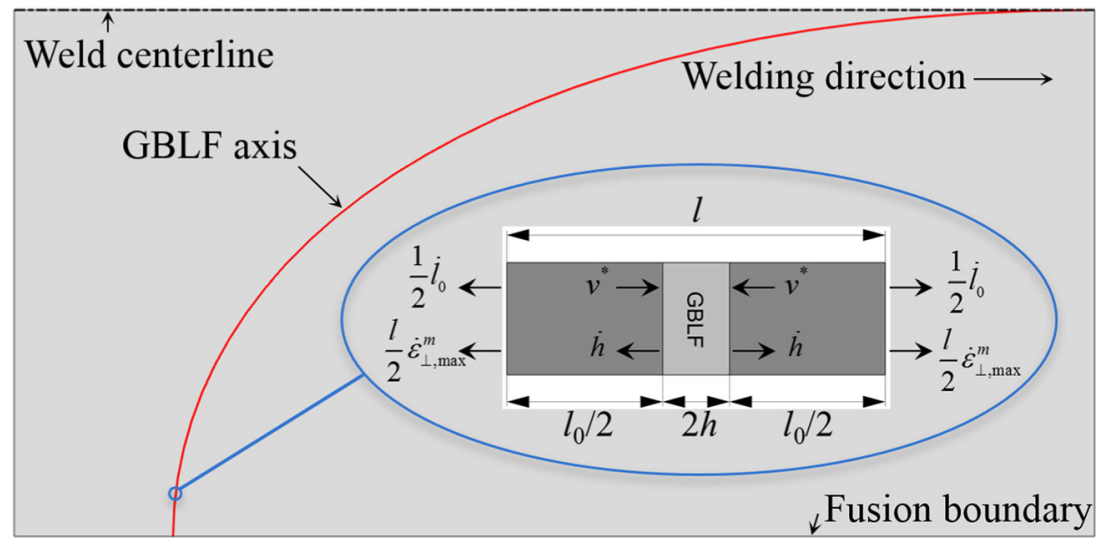

loads. In this case, $l_{0}$ is assumed to be of the same size as the primary dendrite arm spacing. Below the coherent temperature, strains are assumed to localize between the grains and their clusters. The grain cluster formation depends on variations in GBLF thicknesses. Because thin GBLFs can withstand larger tensile loads than thick GBLFs, the deformations will localize in the thicker GBLFs. If a GBLF is very thin, it can coalescence and form a solid grain boundary, GB. The temperature when this occurs depends on the GB force, which in turn depends on the GB misorientation angle. If the misorientation angle is small, the GB force is attractive and coalescence will occur as soon as the opposite solid-liquid interfaces come in contact. However, if the misorientation angle is large, the GB force is repulsive and undercooling is required for coalescence to occur. Rappaz et al. [14] have showed that the required undercooling for GB coalescence of a pure metal is given by

$\Delta T_{b}=\frac{\gamma_{g b}-2 \gamma_{s l}}{\Delta S_{f} \delta}$

where $\gamma_{g b}$ is the GB energy, $\gamma_{s l}$ is the solid-liquid interfacial energy, $\Delta S_{f}$ is the volumetric entropy of fusion, and $\delta$ is the thickness of the diffuse solid-liquid interface. $\gamma_{g b}$ depends on the misorientation angle, and for small misorientation angles, $\gamma_{g b}$ is smaller than $2 \gamma_{s l}$, which result in $\Delta T_{b}<0$ in Eq. 13. Therefore, no undercooling is required for GB coalescence to occur. However, if the misorientation angle is large, $\gamma_{g b}$ is larger than $2 \gamma_{s l}$, which results in $\Delta T_{b}>0$ in Eq. 13, and undercooling is required for GB coalescence to occur.

The variations in GBLF thicknesses, and that GB coalescence depends on the GB misorientation angle, will lead to formation of grain clusters in the solidifying weld metal, i.e., clusters of grains separated by thicker liquid films. When these clusters start to form depends on the temperature. In this study, we assume that all mechanical macroscopic strain localizes between such grain clusters when the temperature is close to the solidus temperature. $l_{0}$ at $T_{s}$ is therefore assumed to be of the same size as the size of a grain cluster. The grain cluster size is not known. In this study we assume it to be proportional to the primary dendrite arm spacing, given by

$l_{0}\left(T_{s}\right)=C_{2} \lambda_{1}$

where $C_{2}$ is a calibration constant that is determined by inverse modeling of a experimental Varestraint test with threshold agumeted strain for crack initiation, which is described in part III of this study [8].

We have now estimated the values of $l_{0}$ at the temperatures $T_{l}, T_{c}$, and $T_{s}$. At temperatures between these values, it is assumed to vary linearly. Figure 5 shows $l_{0}$ as function of the temperature for alloy 718 when $\lambda_{1}=20 \mu \mathrm{m}$. At $T_{s}, l_{0}=0.8 \mathrm{~mm}$ in the Figure, which was obtained by inverse modeling to a Varestraint test with $0.4 \%$ augmented strain, see part III.

\subsubsection{Initial condition}

The initial value of $h$ must be known in order to integrate (9). We assume that $h$ has the same value as the undeformed thickness $h_{0}$ when the GBLF is first formed. If $t_{\text {start }}$ is the time of a given point on the GBLF axis when the temperature drops below the liquidus temperature, the above initial condition can be written as

$h\left(t_{\text {start }}\right)=h_{0}\left(t_{\text {start }}\right)$

\subsection{Liquid pressure model}

The GBLF pressure is determined by assuming that the liquid flow in a GBLF only occurs in the direction of the grain growth, i.e., parallel to the GBLF axis, and that it is governed by Stokes flow at lower fractions of liquid and by Darcy's law at higher fractions of liquid. How the GBLF pressure is computed from those assumptions is shown below. 
Fig. $5 l_{0}$ as a function of temperature for alloy 718 with $\lambda_{1}=20 \mu \mathrm{m}$

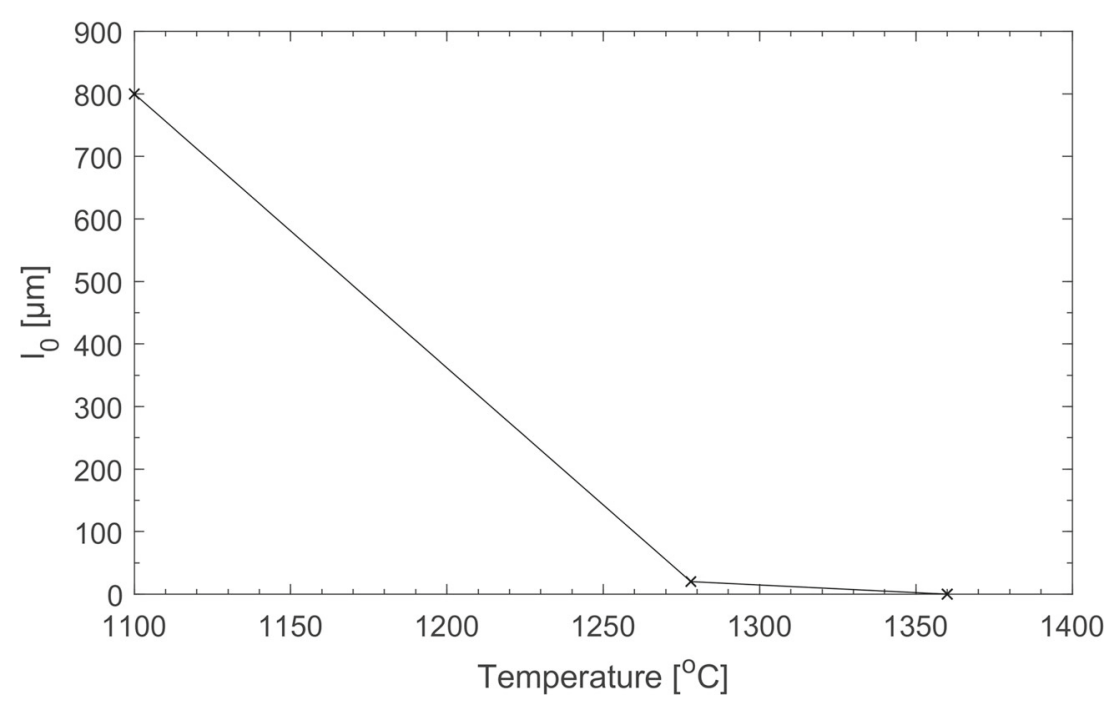

\subsubsection{Liquid flow through a volume element of a GBLF}

Let $\mathbf{v}$ be the liquid velocity field in a given GBLF and assume that the flow is incompressible:

$\nabla \cdot \mathbf{v}=0$

Consider a cross-section volume element of the GBLF, as shown in Fig. 6. Let $x^{\prime} y^{\prime} z^{\prime}$ be a local Cartesian coordinate system such that the $x^{\prime}$-coordinate is tangent to the GBLF axis and the $y^{\prime}$-coordinate is normal to the GBLF axis, see Fig. 6. By integrating (16) over the volume element in Fig. 6, and using the divergence theorem, gives

$\int_{V} \nabla \cdot \mathbf{v} d V=\int_{\partial V} \mathbf{n} \cdot \mathbf{v} d S=0$

where $V$ and $\partial V$ are the volume and boundary of the volume element, respectively. $\mathbf{n}$ is the outward unit normal to the boundary of the volume element. The second integral in Eq. 17 can be split into two parts: one over the solid-liquid interfaces, $\partial V_{s l}$, and one over the cross-section parts, $\partial V_{l}$, of the liquid film:

$\int_{\partial V} \mathbf{n} \cdot \mathbf{v} d S=\int_{\partial V_{s l}} \mathbf{n} \cdot \mathbf{v} d S+\int_{\partial V_{l}} \mathbf{n} \cdot \mathbf{v} d S$

As was previously stated, we assume that the flow is dominated by that in the longitudinal direction of the GBLF, i.e., in the columnar direction of the grains, and is independent of the transverse $z^{\prime}$ direction. This assumption gives the velocity field

$\mathbf{v}=v\left(x^{\prime}, y^{\prime}\right) \mathbf{e}_{x^{\prime}}$

By inserting (19) into the integral over $\partial V_{s l}$ in Eq. 18, it can be rewritten as

$\int_{\partial V_{s l}} \mathbf{n} \cdot \mathbf{v} d S=\left(v_{l}^{*}+\left(v_{s l}^{+}+v^{*}\right)\right) \Delta x^{\prime} \Delta z^{\prime}+\left(v_{l}^{*}-\left(v_{s l}^{-}-v^{*}\right)\right) \Delta x^{\prime} \Delta z^{\prime}$ where $v_{s l}^{+}$and $v_{s l}^{-}$are the velocities of the two opposing solid-liquid interfaces, as shown in Fig. 6. $v_{l}^{*}$ is the liquid flow caused by solidification shrinkage, which is given by [4]

$v_{l}^{*}=\beta v^{*}$

where $\beta$ is the solidification shrinkage factor and $v^{*}$ is the solidification velocity, which is given by Eq. 8. Note that we have neglected the liquid flow through the solid-liquid interfaces in Eq. 20. This assumption is discussed in the end of Section 3.3.

By inserting (19) into the integral over $\partial V_{l}$ in Eq. 18, it can be expressed as

$\int_{\partial V_{l}} \mathbf{n} \cdot \mathbf{v} d S=\left(2 h^{+} \bar{v}^{+}-2 h^{-} \bar{v}^{-}\right) \Delta z^{\prime}$

where $h^{+}$and $h^{-}$are the half GBLF thicknesses, and $\bar{v}^{+}$ and $\bar{v}^{-}$are the average normal liquid velocities at the cross sections in the GBLF axis direction, as shown in Fig. 6.

The term $v_{s l}^{+}-v_{s l}^{-}$in Eq. 20 is the relative normal velocity term of the two opposing solid-liquid interfaces of the GBLF, and can therefore be determined from the GBLF thickness rate as:

$v_{s l}^{+}-v_{s l}^{-}=2 \frac{d h}{d t}$

Combining (18) and (20)-(23) and taking the limit $\Delta x^{\prime} \rightarrow$ 0 , we obtain

$\frac{d(h \bar{v})}{d x^{\prime}}=-(1+\beta) v^{*}-\frac{d h}{d t}$

Equation 24 correlates $\bar{v}, d h / d t$, and $v^{*} . \bar{v}$ is determined for two different cases: at low and high fractions of liquid. This is done as follows. 
Fig. 6 Cross-section volume element of a GBLF

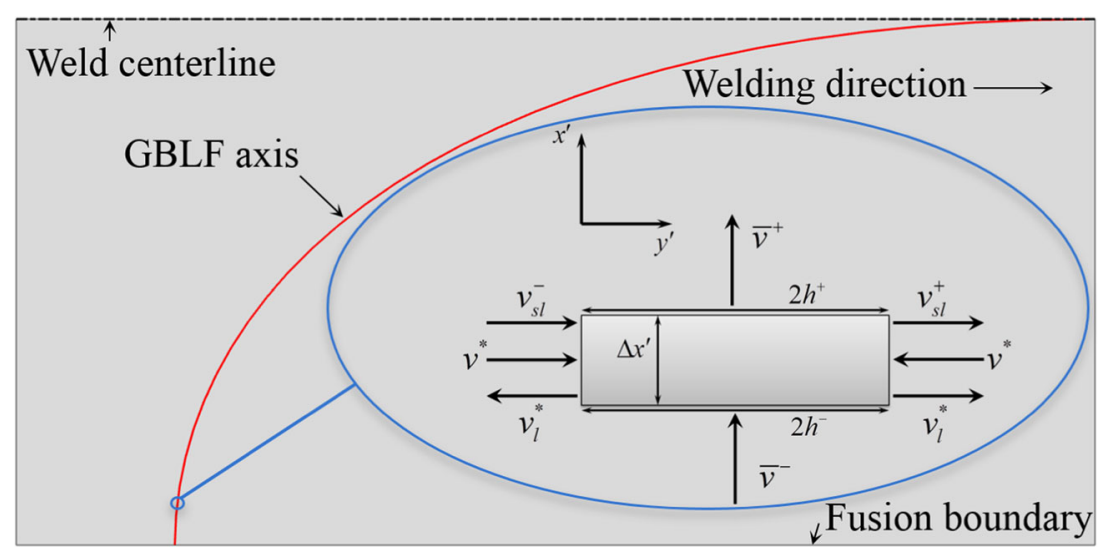

\subsubsection{Liquid flow at low fractions of liquid}

At low fractions of liquid, the secondary dendrite arms of individual dendrites are almost fully coalescenced. Thus, liquid flow around secondary arms is difficult and the flow is therefore assumed to be restricted to the grain boundaries. Furthermore, at low fractions of liquid, large grain cluster may have formed such that the flow is further restricted to the GBLFs between these grain clusters. In this study, we assume that the flow at low fractions of liquid occurs between large grain cluster as wide liquid films. Moreover, the liquid is assume to be Newtonian and the flow is assumed to occur at low Reynolds numbers such that the inertial forces are small compared with the viscous forces. The flow can then be approximated with Stokes equations [15]:

$\mu \nabla^{2} \mathbf{v}-\nabla p=\mathbf{0}$

where $p$ is the liquid pressure and $\mu$ is the dynamic viscosity. Substituting the velocity field in Eq. 19 into (25) gives

$\mu\left(\frac{\partial^{2} v}{\partial x^{\prime 2}}+\frac{\partial^{2} v}{\partial y^{\prime 2}}\right)-\frac{\partial p}{\partial x^{\prime}}=0$

The first term on the left-hand side is much smaller than the other terms, which is shown by the following scaling. Let us introduce the normalized variables

$\tilde{x}^{\prime}=\frac{x^{\prime}}{L_{c}}, \quad \tilde{y}^{\prime}=\frac{y^{\prime}}{2 h}, \quad \tilde{v}=\frac{v}{v_{c}}, \quad \tilde{p}=\frac{p}{p_{c}}$

where $L_{c}$ is the characteristic length of a GBLF, $v_{c}$ is a characteristic liquid velocity, and $p_{c}$ is a characteristic liquid pressure. By inserting these variables into Eq. 26, it can be written as

$\frac{\mu v_{c}}{L_{c}^{2}} \frac{\partial^{2} \widetilde{v}}{\partial \widetilde{x}^{\prime 2}}+\frac{\mu v_{c}}{4 h^{2}} \frac{\partial^{2} \widetilde{v}}{\partial \widetilde{y}^{\prime 2}}-\frac{p_{c}}{L_{c}} \frac{\partial \widetilde{p}}{\partial \widetilde{x}^{\prime}}=0$
Characteristic values for this study are (i.e., for TIG welding of a 3-mm-thick plate of alloy 718 with a welding speed of $1 \mathrm{~mm} / \mathrm{s}$, see part III):

$$
\begin{aligned}
& L_{c} \sim 10^{-3} \mathrm{~m}, v_{c} \sim 10^{-3} \mathrm{~m} / \mathrm{s}, \mu \sim 10^{-2} \mathrm{~m}^{2} / \mathrm{s}, \\
& h \sim 10^{-6} \mathrm{~m}, p_{c} \sim-10^{5} \mathrm{~Pa}
\end{aligned}
$$

Inserting these values into the coefficients of Eq. 28 then gives

$\frac{p_{c}}{L_{c}} \sim-10^{8}, \frac{\mu v_{c}}{L_{c}^{2}} \sim 10^{1}, \frac{\mu v_{c}}{4 h^{2}} \sim 10^{7}$

The coefficient in front of the $\partial^{2} \widetilde{v} / \partial \widetilde{x}^{\prime 2}$ term is several orders of magnitude smaller than the other two. Thus, the $\partial^{2} v / \partial x^{\prime 2}$ term in Eq. 26 can be neglected, which then reduces to

$\mu \frac{\partial^{2} v}{\partial y^{\prime 2}}-\frac{\partial p}{\partial x^{\prime}}=0$

By integrating (31) twice across the liquid film, and applying the non-slip boundary conditions $v\left(y^{\prime}=-h\right)=$ $v\left(y^{\prime}=h\right)=0$, gives the solution for a Poiseuille flow between parallel plates:

$v=\frac{1}{2 \mu} \frac{\partial p}{\partial x^{\prime}}\left(y^{\prime 2}-h^{2}\right)$

The relative parallel velocity component between the two opposing solid-liquid interfaces has been neglected. Poiseuille flow between parallel plates has been used by Sistaninia et al. [16] in their granular model to compute the pressure in GBLFs between globular grains.

The mean velocity across the GBLF can be obtained from Eq. 32 as

$\bar{v}=-\frac{h^{2}}{3 \mu} \frac{d p}{d x^{\prime}}$

Substituting (33) into Eq. 24 finally gives

$\frac{d}{d x^{\prime}}\left(\frac{h^{3}}{3 \mu} \frac{d p}{d x^{\prime}}\right)=\frac{d h}{d t}+(1+\beta) v^{*}, \quad f_{l} \leq 0.1$

This is Reynolds equation (without relative parallel motion of the two opposing interfaces of the GBLF). 


\subsubsection{Liquid flow at high fractions of liquid}

At high fractions of liquid, flow can occur around the secondary dendrite arms. Therefore, the Poiseuille parallel plate flow, which was previously used for low fractions of liquid, is not good in this case. Instead, we assume that the flow now more resembles a porous flow governed by Darcy's law [4]. The average liquid velocity $\bar{v}$ in Eq. 24 can then be approximated as

$\bar{v}=-\frac{K_{\|}}{f_{l}^{*} \mu} \frac{d p}{d x^{\prime}}$

where $K_{\|}$is the longitudinal permeability of the GBLF in the axial direction of the GBLF, and $f_{l}^{*}$ is an effective fractions of liquid for the GBLF, see Eq. (39). Heinrich and Poirier [6] have estimated the columnar interdendritic longitudinal permeability as

$K_{\|}= \begin{cases}3.75 \times 10^{-4} f_{l}^{2} d_{1}^{2}, & f_{l} \leq 0.65 \\ 2.05 \times 10^{-7}\left[\frac{f_{l}}{1-f_{l}}\right]^{10.739} d_{1}^{2}, & 0.65<f_{l} \leq 0.75 \\ 0.074\left[\log \left(\frac{1}{1-f_{l}}\right)+0.01-f_{l}-0.5 f_{l}^{2}\right] d_{1}^{2}, & 0.75<f_{l} \leq 1.0\end{cases}$

and the transverse columnar dendritic permeability as

$$
K_{\perp}= \begin{cases}1.09 \times 10^{-3} f_{l}^{3.32} d_{1}^{2}, & f_{l} \leq 0.65 \\ 4.04 \times 10^{-6}\left[\frac{f_{l}}{1-f_{l}}\right]^{6.7336} d_{1}^{2}, & 0.65<f_{l} \leq 0.75 \\ \left(-6.49^{-2}+5.43^{-2}\left[\frac{f_{l}}{1-f_{l}}\right]^{0.25}\right) d_{1}^{2}, & 0.75<f_{l} \leq 1.0\end{cases}
$$

where $d_{1}$ is the primary dendrite arm distance. The above permeabilities were obtained with regression analysis of empirical data when $f_{l} \leq 0.65$ and by numerical simulations when $f_{l}>0.65$ [6].

To estimate the GBLF permeability, we assume it to be equivalent to the above permeability in Eq. 36, but with modified values of $d_{1}$ and $f_{l}$ in order to account for the increase in permeability that occurs when deformation is localized in the GBLF. The modified $d_{1}$ and $f_{l}$ are approximated as follows. Two dendrites on the opposite sides of the solid-liquid interfaces of a GBLF, with an initial spacing of $\lambda_{1}$, will have the spacing

$d_{1}^{*}=\lambda_{1}+2 h-2 h_{0}$

when the GBLF thickness is $2 h$. Consider an arrangement of columnar dendrites situated on a square grid with spacing $\lambda_{1}$. Now, consider the same arrangement with the same dendrites, but with the grid spacing $d_{1}^{*}$. The fraction of liquid for this system can then be written as

$f_{l}^{*}=1-\frac{\lambda_{1}^{2}\left(1-f_{l}\right)}{d_{1}^{* 2}}$ where $f_{l}$ is the fraction of liquid of the system with the grid spacing $\lambda_{1}$. We now assume that the GBLF permeability is the same as in Eq. 36, but with $d_{1}$ and $f_{l}$ given by Eqs. 38 and 39 , respectively, in order to account for the change in permeability caused by deformation.

By inserting (35) into Eq. 24, the following equation for the pressure in the GBLF at high fractions of liquid is obtained

$\frac{d}{d x^{\prime}}\left(\frac{K_{\|} h}{\mu f_{l}^{*}} \frac{d p}{d x^{\prime}}\right)=\frac{d h}{d t}+(1+\beta) v^{*}, \quad f_{l}>0.1$

where $K_{\|}$is given by Eq. 36 with $d_{1}$ and $f_{l}$ given by Eqs. 38 and 39 , respectively.

The cross permeability in Eq. 37 is not used in any flow calculations in this study, it is just used to compute the ratio between $K_{\perp}$ and $K_{\|}$in order to discuss the effect of neglecting the transverse flow through the solid-liquid interface of the GBLF (see Section 3.3). To compute this ratio, the permeability of the GBLF for $f_{l} \leq 0.1$ (when the flow is governed by the Poiseuille flow) must be known. This is obtained by setting the right-hand side of Eq. 35 equal to that of Eq. 33 and solving for $K_{\|}$, which gives

$K_{\|}=\frac{h^{2} f_{l}^{*}}{3}, \quad f_{l} \leq 0.1$

\subsubsection{Pressure integration}

The GBLF pressure is now determined as follows. Let $s$ be a curved coordinate along the GBLF axis with origin at the fusion boundary (Fig. 7). The pressure in the GBLF is computed by integrating (34) and (40) along $s$. Since the GBLF thickness is much smaller than the radius of curvature of the GBLF axis, the influence of the curvature in the integration is neglected. For a given time, the location of the start of the integration, $s=s_{T_{s}}$, is at the intersection of the GBLF axis with the $T_{s}$ isotherm. The location of the end of the integration, $s=s_{T_{l}}$, is at the intersection of the GBLF axis with the $T_{l}$ isotherm, as shown in Fig. 7. Note that $s=s_{T_{s}}$ and $s=s_{T_{l}}$ move with time when the solidification progresses.

The transition point between Poiseuille flow and Darcy flow is set as the location where the fraction of liquid is $f_{l}=$ 0.1 . As was previously stated, the Poiseuille flow model is associated with the part of the GBLF whose interfaces are bounded by grain clusters. Vernede [17] has developed a 2D granular numerical model for flow simulation in the mushy zone. He used that model to show, for an aluminum alloy that solidifies with granular grains, that grain clusters start to form at a rapid rate when the fraction of liquid is less than approximately $f_{l}=0.1$. This value of $f_{l}$ was used as the transition point between the Poiseuille and Darcy flows in this study. We define $s=s_{\text {trans }}$ as the location of this transition point at a given time. It is determined from the 
Fig. 7 Schematic of the integration path for the GBLF pressure

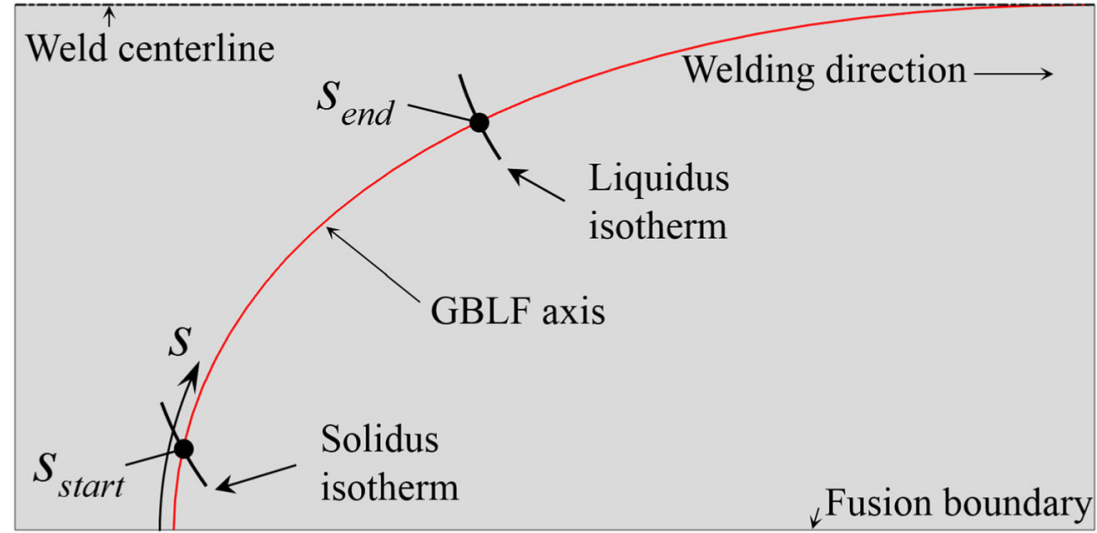

intersection of the GBLF axis with the temperature isotherm corresponding to $f_{l}=0.1$.

The GBLF pressure is now integrated as follows. First, Reynolds equation (34) is integrated between $s_{T_{s}}$ and $s_{\text {trans }}$ with the boundary condition for $d p / d s$ at $s_{T_{s}}$, which is given in the next section. Then, Eq. 40 is integrated twice between $s_{\text {trans }}$ and $s_{T_{1}}$. In the first integration, the following boundary condition at $s_{\text {trans }}$ is used, which ensures that the liquid flow $(\bar{v})$ is continuous at the transition point:

$$
\frac{d p\left(s=s_{\text {trans }}^{+}\right)}{d s}=\left.\left(\frac{h^{2} f_{l}^{*}}{3 K_{\|}} \frac{d p}{d s}\right)\right|_{s=s_{\text {trans }}}
$$

where $d p\left(s=s_{\text {trans }}\right) / d s$ can be obtained from the first integration of the Reynolds equation. The boundary condition in Eq. 42 is obtained by combining (33) and (35). In the second integration of Eq. 40, the boundary condition $p\left(s_{T_{l}}\right)$ is used, which is defined in the next section. The value of $p\left(s_{\text {trans }}\right)$ can now be computed from this second integration and is used in the second integration of the Reynold equation (34). The pressure in the GBLF can then finally be written as

$p(s)= \begin{cases}p\left(s_{\text {trans }}\right)-\int_{s}^{s_{\text {trans }}} F_{R}\left(s^{\prime}\right) d s^{\prime}, & s \leq s_{\text {trans }} \\ p\left(s_{T_{l}}\right)-\int_{s}^{s T_{l}} F_{D}\left(s^{\prime}\right) d s^{\prime}, & s>s_{\text {trans }}\end{cases}$

where

$$
F_{R}(s)=\frac{3 \mu}{h^{3}}\left[\int_{s_{T_{l}}}^{s}\left(\frac{d h}{d t}+(1+\beta) v^{*}\right) d s^{\prime}+\left.\left(\frac{h^{3}}{3 \mu} \frac{d p}{d s}\right)\right|_{s=T_{s}}\right]
$$

and

$$
F_{D}(s)=\frac{\mu f_{l}^{*}}{K_{\|} h}\left[\int_{s_{\text {trans }}}^{s}\left(\frac{d h}{d t}+(1+\beta) v^{*}\right) d s^{\prime}+\left.\left(\frac{10 K_{\|} h}{\mu} \frac{d p}{d s}\right)\right|_{s=s_{\text {trans }}}\right]
$$

The variables $v^{*}$ and $h$ in Eqs. 44 and 45 are given by Eqs. 8 and 10, respectively. The pressure in Eq. 43 is solved by numerical integration. The integrands are evaluated from temperature and macroscopic strain data from a computational welding mechanics model of the welding process (see part III [8]). These data are evaluated from the same Lagrangian sample points that were used to trace out the GBLF axis, which was discussed previously in Section 2.4.1.

\subsubsection{Boundary conditions}

The boundary conditions $p\left(s=s_{T_{l}}\right)$ and $d p\left(s=s_{T_{s}}\right) / d s$ are used to evaluate the pressure in Eq. 43. These are defined as follows. At the location of intersection of the GBLF axis with the $T_{l}$ isotherm, the GBLF pressure is assumed to be the same as the atmospheric pressure, hence

$p\left(s_{T_{l}}\right)=p_{a t m}$

At $s_{T_{s}}$, i.e., at the intersection of the GBLF axis with the $T_{s}$ isotherm, $d p\left(s_{T_{s}}\right) / d s$ can be expressed as

$$
\frac{d p\left(s_{T_{s}}\right)}{d s}=\left.\left(\frac{3 \mu \beta}{h^{2}} \frac{d s_{T_{s}}}{d t}\right)\right|_{s=T_{s}}
$$

where $d s_{T_{s}} / d t$ is the solidification velocity at $s_{T_{s}}$ in the direction of the GBLF axis. Note that $d p / d s$ is related to the liquid flow in the GBLF according to Eq. 33. Thus, the boundary condition in Eq. 47 corresponds to the pressure drop at the end of the liquid film due to the flow caused by solidification shrinkage of the remaining liquid at the end of the GBLF.

\section{Evaluation}

The derived GBLF pressure model was evaluated on Varestraint tests of alloy 718 . The test specimens were prepared from 3.2-mm-thick plates and autogenous TIG welding with a welding speed of $1 \mathrm{~mm} / \mathrm{s}$ was used in the tests. The augmented strain was applied to a test specimen by bending it over a die block when the weld length reached $40 \mathrm{~mm}$. The stroke rate was $10 \mathrm{~mm} / \mathrm{s}$ and welding continued for $5 \mathrm{~s}$ after the start of the bending. The amount of 

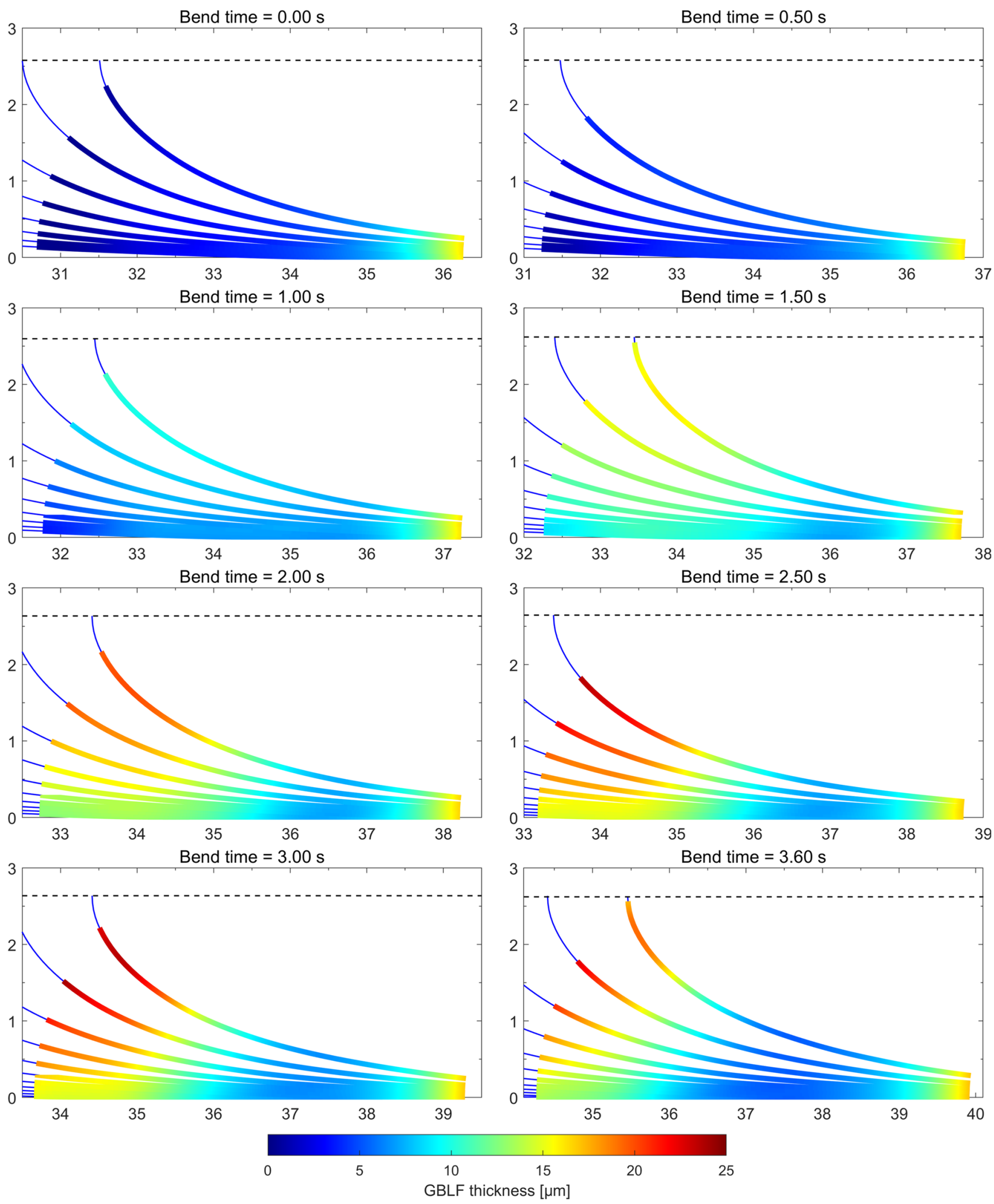

Fig. 8 Evolution of GBLF thickness at the weld surface for a Varestraint test with $1.1 \%$ augmented strain. Only the left part of the symmetric weld is shown. The time in the plots represents the elapsed time since the start of the bending. The abscissa and ordinate represent the distance from the weld start and weld centerline, respectively 

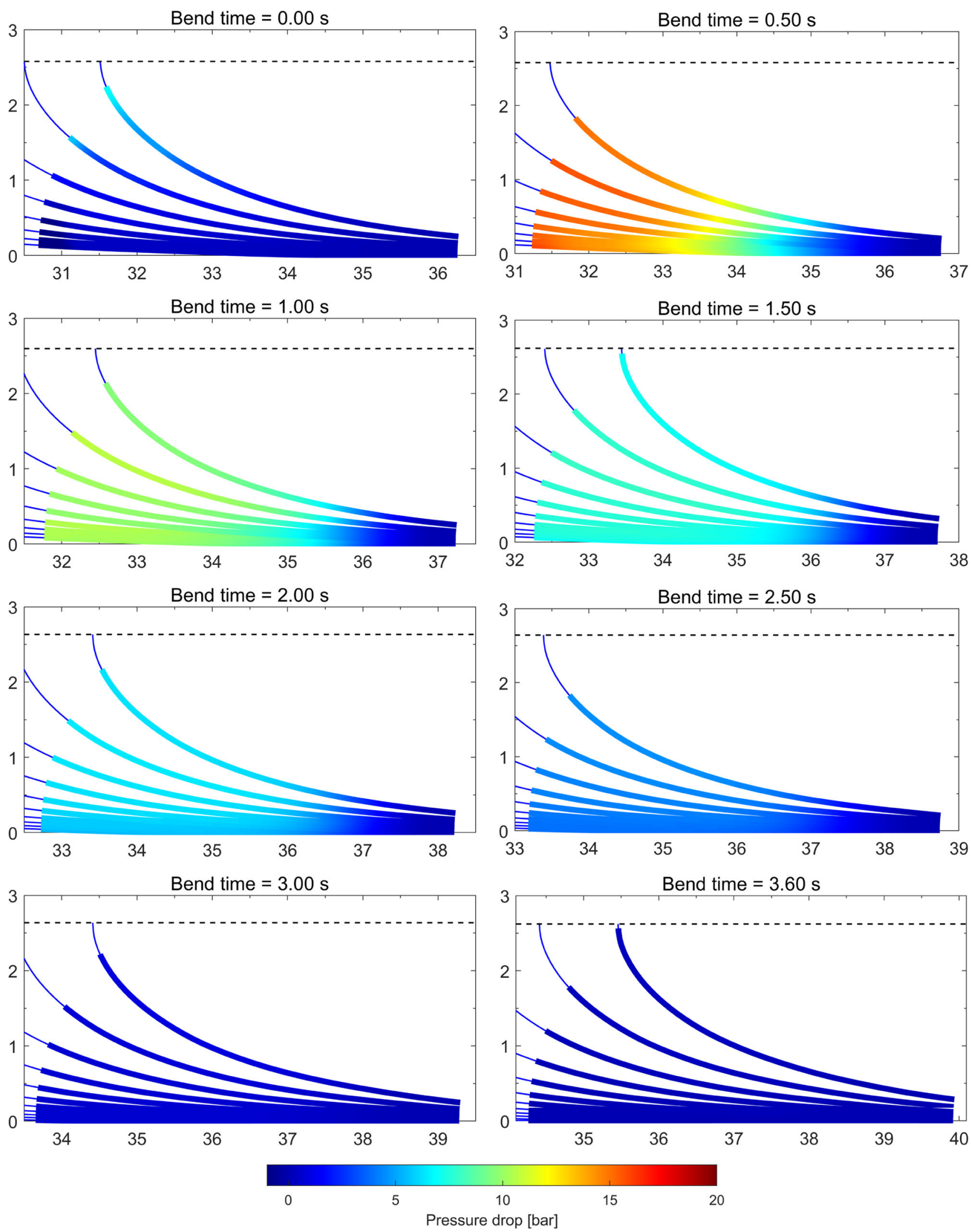

Fig. 9 Evolution of GBLF pressure drop at the weld surface for a Varestraint test with $1.1 \%$ augmented strain. Only the left part of the symmetric weld is shown. The time in the plots represents the elapsed time since the start of the bending. The abscissa and ordinate represent the distance from the weld start and weld centerline, respectively 

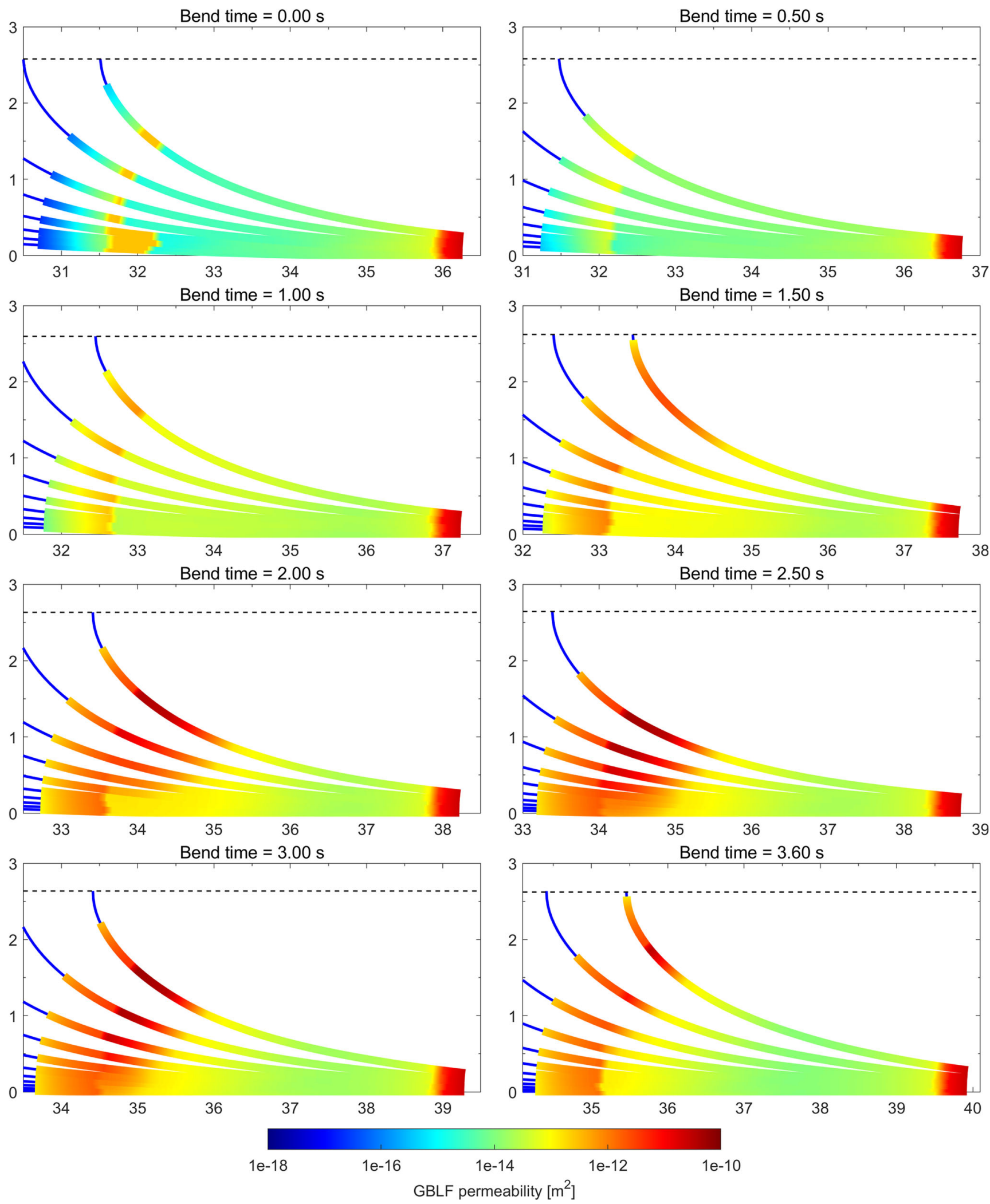

Fig. 10 Evolution of longitudinal permeability at the weld surface for a Varestraint test with $1.1 \%$ augmented strain. Only the left part of the symmetric weld is shown. The time in the plots represents the elapsed time since the start of the bending. The abscissa and ordinate represent the distance from the weld start and weld centerline, respectively 


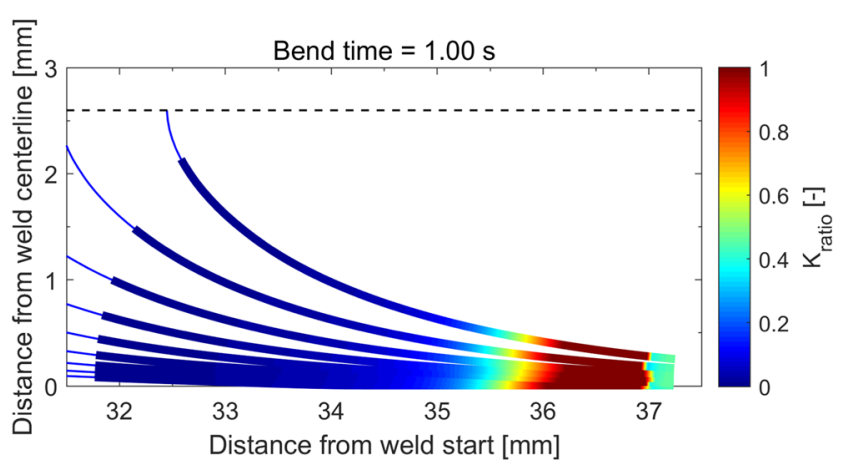

Fig. 11 Ratio between transverse and longitudinal permeability at $1 \mathrm{~s}$ of bend time, evaluated at the weld surface for a Varestraint test with $1.1 \%$ augmented strain

augmented strain was controlled by the radius of the die block. More details about the Varestraint test can be found in part III of this work [8].

The evolution of the pressure drop, thickness, and permeability of GBLFs in the Varestraint test with $1.1 \%$ augmented strain, as predicted by the developed model in this paper, is shown below. These quantities were evaluated on GBLF axes that are located at the surfaces of the weld. The $x$ and $y$ coordinates in the below plots represent the distances from the weld start and weld centerline, respectively. The welding direction is from the left to right. The blue lines in the plots represent the computed GBLF axes. They are separated approximately $1 \mathrm{~mm}$ at the fusion boundary, such that they together cover the region with the highest crack susceptibility. This region is located 31 to 35 $\mathrm{mm}$ from the weld start. The apex of the die block is located $40 \mathrm{~mm}$ from the weld start [8]. Only GBLFs whose axis intersects the solidus isotherm inside the fusion zone were considered. GBLFs that extend into the partially melted zone will be considered in future work. The bend time in the below plots represents the elapsed time from the initiation of bending. The temperature field and macroscopic strain field, which are required to evaluate the above quantities, are obtained from the computational welding mechanics model which is described in part III of this work [8].

\subsection{GBLF thickness}

Figure 8 shows the evolution of GBLF thickness for a Varestraint test with $1.1 \%$ augmented strain. Only the left part of the symmetric weld is shown. The full bending takes $3.6 \mathrm{~s}$ to complete. When the bending starts, $2 h$ is approximately equal to $2 h_{\min }$ at $s=s_{T_{s}}$, as can be seen from the Figure. However, with increasing bending, deformations start to localize in the GBLFs.
The rate of deformation is highest for the GBLFs that are directed perpendicular to the bending direction, i.e., directed perpendicular to the weld centerline. The rate of deformation is also higher at the ends of the GBLFs ( $s=s_{T_{s}}$ ) compared to the starts of the GBLFs $\left(s=s_{T_{l}}\right)$ because the strain localization is largest at the GBLF end. A maximum value of $2 h=20 \mu \mathrm{m}$ is reached approximately $3 \mathrm{~s}$ after the bending started. This shows that the $1.1 \%$ augmented strain that is applied in the Varestraint test is strongly localized in GBLFs. The maximum values of $2 h$ for Varestraint tests with $0.4 \%$ and $0.8 \%$ augmented strains are approximately 7 and $15 \mu \mathrm{m}$, respectively. For more details on the variation of $2 h$ with time for Varestraint tests with different augmented strains, please refer to the appended animations.

\subsection{GBLF pressure drop}

Figure 9 shows the evolution of the GBLF pressure drop $\left(\Delta p=p_{a t m}-p\right)$ for a Varestraint test with $1.1 \%$ augmented strain. $\Delta p$ reaches a maximum approximately $0.30 \mathrm{~s}$ after the bending started. Thereafter, it starts to decrease, even though $2 h$ continues to increase, as can be seen in Fig. 8 . This is because the deformation increases the permeability, which is shown in Fig. 10. The increase in permeability simplifies liquid feeding, which results in a decrease in the pressure drop. Note that the pressure drop is almost zero at the end of the bending (Fig. 9). $\Delta p$ in the $0.4 \%$ and $0.8 \%$ tests evolves with the same trends as in the $1.1 \%$ test (see the appended animations).

\subsection{GBLF permeability}

Figure 10 shows the evolution of the longitudinal permeability (36 and 41) for a Varestraint test with $1.1 \%$ augmented strain. As can be seen from the plots, $K_{\|}$increases several orders at the GBLF ends when deformation increases the GBLF thickness.

One major assumption in this work is that the liquid flow in a GBLF is solely confined to the GBLF such that no liquid can flow across the solid-liquid interfaces of the GBLF. This is a rough approximation. However, when $f_{l}$ goes to zero, the ratio between the transverse and longitudinal permeability also goes to zero. This is shown in Fig. 11 for a Varestraint test with $1.1 \%$ augmented strain, at $1 \mathrm{~s}$ of bend time. It can be seen in this figure and Fig. 9 that the largest pressure drops occur in the part of the film where this ratio is less than 0.1 . Thus, the assumption of no liquid flow through the solid-liquid interfaces of the GBLF seems valid in the part of the GBLF where the largest pressure drop occurs, which is also where cracking occurs. 


\section{Conclusions}

A solidification cracking criterion was introduced in part I of this work. In order to evaluate this criterion for estimating the crack susceptibility, the GBLF pressure and GBLF thickness must be known. In this paper, we introduce a model for estimating these quantities in a columnar dendritic microstructure. This model contains a submodel that determines an axis of a GBLF from the temperature field of a computational welding mechanics model. The liquid flow in the GBLF is assumed to be along the direction of this axis. The solidification of the liquid in the GBLF is governed by the Scheil-Gulliver model. A submodel is used to compute the GBLF thickness from the macroscopic mechanical strain field of the computational welding mechanics model, where a temperature-dependent length scale is used to localize the macroscopic mechanical strain to the GBLF. At the liquidus temperature, this length is zero; at the coherent temperature, it is equal to the primary dendrite arm spacing; and at solidus, it is the same as the diameter of a grain cluster, which is a calibration constant. Between these temperatures, it is assumed to vary linearly. The liquid flow within the GBLF is assumed to be governed by a combination of Poiseuille and Darcy flows. For the part of the GBLF with less than 0.1 fractions of the liquid, the flow is a Poiseuille flow. For the remaining part, the flow is a Darcy flow. The permeability used for the Darcy flow is derived from empirical data and numerical simulations and depends on the deformation of the GBLF.

The model has been evaluated on Varestraint tests of alloy 718. The evolution of the GBLF thickness, GBLF pressure drop, and GBLF permeability was studied.

Acknowledgments The authors are thankful to Rosa Maria Pineda Huitron from the Material Science Department at Luleå Technical University for the help with evaluating the experimental Varestraint tests.

Funding information This study was financially supported by the NFFP program, run by Swedish Armed Forces, Swedish Defence Materiel Administration, Swedish Governmental Agency for Innovation Systems, and GKN Aerospace (project numbers: 2013-01140 and 2017-04837).

Open Access This article is distributed under the terms of the Creative Commons Attribution 4.0 International License (http:// creativecommons.org/licenses/by/4.0/), which permits unrestricted use, distribution, and reproduction in any medium, provided you give appropriate credit to the original author(s) and the source, provide a link to the Creative Commons license, and indicate if changes were made.

\section{Appendix}

The undercooling models that were used in Section 2.2 are given in this appendix.
Constitutional undercooling model Foster et al. [9] used the following model to estimate the constitutional undercooling for alloy 718

$\Delta T_{C}=\sum_{i=1}^{n}\left(C_{0}^{i} m_{0}^{i}-C_{l}^{* i} m_{R_{L}}^{i}\right)$

Here, $C_{0}^{i}$ is the nominal concentration of the $i$ th element in the liquid phase, $m_{0}^{i}$ is the equilibrium liquidus slope, $m_{R_{L}}^{i}$ is the velocity-dependent liquidus slope, and $C_{l}^{* i}$ is the liquid concentration of the $i$ th element at the dendrite tip. $C_{l}^{* i}$ is determined by the following expression

$C_{l}^{* i}=\frac{C_{0}^{i}}{1-\left(1-k_{R_{L}}^{i}\right) \operatorname{Iv}\left(\mathrm{Pe}_{C}^{i}\right)}$

where $k_{R_{L}}^{i}$ is the velocity-dependent partitioning coefficient, given by:

$k_{R_{L}}^{i}=\frac{k_{0}^{i}+a_{0} R_{L} / D_{l}^{i}}{1+a_{0} R_{L} / D_{l}^{i}}$

In the above expression, $k_{0}^{i}$ is the equilibrium partition coefficient of the $i$ th element, $a_{0}$ is the characteristic diffusion distance, $R_{L}$ is the growth velocity of the dendrite tip, and $D_{l}^{i}$ is the solute diffusivity of element " $i$." In Eq. 49, $\mathrm{Pe}_{C}^{i}$ is the solutal Peclet number, defined by

$\mathrm{Pe}_{C}^{i}=\frac{R_{L} R_{t i p}}{2 D_{l}^{i}}$

and $\operatorname{Iv}\left(\mathrm{Pe}_{C}^{i}\right)$ is the Ivantsov function, given by:

$\operatorname{Iv}\left(\mathrm{Pe}_{C}^{i}\right)=\mathrm{Pe}_{C}^{i} \exp \left(\mathrm{Pe}_{C}^{i}\right) \mathrm{E}_{1}\left(\mathrm{Pe}_{C}^{i}\right)$

where $E_{1}$ is the exponential integral:

$\mathrm{E}_{1}\left(\mathrm{Pe}_{C}^{i}\right)=\int_{\mathrm{Pe}_{C}^{i}}^{\infty} \frac{\exp (-s)}{s} d s$

The $m_{R_{L}}^{i}$ term in Eq. 49 is defined as

$m_{R_{L}}^{i}=m_{0}^{i}\left[\frac{1-k_{R_{L}}^{i}\left(1-\ln \left(k_{R_{L}}^{i} / k_{0}^{i}\right)\right)}{1-k_{0}^{i}}\right]$

The curvature of the dendrite tip and the thermal gradient at the tip are related by the interface instability criteria:

$4 \pi^{2} \Gamma+G_{L} R_{t i p}^{2}+2 R_{t i p} \sum_{i=1}^{n}\left(m_{R_{L}}^{i} \operatorname{Pe}_{C}^{i}\left(1-k_{R_{L}}^{i}\right) C_{l}^{* i} \xi_{C}^{i}\right)=0$

where $\Gamma$ is the Gibbs-Thompson coefficient and $\xi_{C}^{i}$ is the absolute stability coefficient, given by

$\xi_{C}^{i}=1-\frac{2 k_{R_{L}}^{i}}{2 k_{R_{L}}^{i}-1+\sqrt{1+\left(2 \pi / \mathrm{Pe}_{C}^{i}\right)^{2}}}$ 
Table 1 Parameters used for the undercooling models, from [9]

\begin{tabular}{llllll}
\hline Element ID & Comp. [At. Pct] & $k_{0}^{i}-$ & $m_{0}^{i}\left[\mathrm{~K} \times(\text { At. Pct })^{-1}\right]$ & Parameters & Value \\
\hline $\mathrm{Al}$ & 1.27 & 1.16 & -5.76 & $a_{0}[\mathrm{~m}]$ & $3.0 \times 10^{-10}$ \\
$\mathrm{Cr}$ & 20.09 & 1.07 & -2.63 & $\Gamma[\mathrm{mK}]$ & $3.37 \times 10^{-7}$ \\
$\mathrm{Fe}$ & 18.13 & 1.19 & -1.04 & $D_{l}^{i}\left[\mathrm{~m}^{2} \mathrm{~s}^{-1}\right]$ & $5.0 \times 10^{-9}$ \\
$\mathrm{Mo}$ & 1.77 & 0.65 & -6.35 & \\
$\mathrm{Nb}$ & 3.20 & 0.20 & -14.63 & \\
$\mathrm{Ti}$ & 1.03 & 0.40 & -15.19 & \\
$\mathrm{C}$ & 0.16 & 0.13 & -10.70 & \\
$\mathrm{Ni}$ & 54.01 & 1.02 & 1.04 & \\
$\mathrm{O}$ & 0.04 & 0.25 & -3.66 & \\
\hline
\end{tabular}

For given values of $R_{L}$ and $G_{L}$, and for given values of the parameters $k_{0}^{i}, m_{0}^{i}, a_{0}, \Gamma$, and $D_{l}^{i}$, the only unknown in Eq. 55 is $R_{t i p}$, which can be solved for by a numerical root finder such as the Matlab function fzero. When $R_{t i p}$ is known, $\Delta T_{C}$ in Eq. 48 can finally be determined.

Foster et al. [9] used the thermodynamic software Thermo-Calc to calculate $k_{0}^{i}$ and $m_{0}^{i}$ for the elements in alloy 718, which are reproduced in Table 1 . Due to lack of data, they used the same value for $D_{l}^{i}$ for all the elements.

The $\Delta T_{C}$ curve in Fig. 2 was calculated with the model in Eq. 48 for given values of $R_{L}$ and $G_{L}$, together with the data in Table 1.

Curvature undercooling model Foster et al. [9] calculated the curvature undercooling with the following model

$\Delta T_{R}=\frac{2 \Gamma}{R_{t i p}}$

where $R_{t i p}$ is determined from Eq. 55 . The $\Delta T_{R}$ curve in Fig. 2 is computed with this model together with the $\Gamma$ value in Table 1.

Thermal undercooling The following thermal undercooling model, stated in Dantzig and Rappaz [4], was used to compute $\Delta T_{T}$ in Fig. 2

$\Delta T_{T}=\frac{L_{f}}{c_{p}} \operatorname{Iv}\left(\mathrm{Pe}_{T}\right)$

Here, $L_{f}$ and $c_{p}$ are the latent heat of fusion and the specific heat capacity, respectively. $\mathrm{Pe}_{T}$ is the thermal Peclet number, given by:

$\mathrm{Pe}_{T}=\frac{R_{L} R_{t i p}}{2 \alpha_{l}}$

where $\alpha_{l}$ is the thermal diffusivity of the liquid. The value of $R_{t i p}$ that goes into Eq. 58 was computed from Eq. 55 . $L_{f}=241 \times 10^{3} \mathrm{Jkg}^{-1} \mathrm{~K}^{-1}, c_{p}=720 \mathrm{Jkg}^{-1} \mathrm{~K}^{-1}$, and $\alpha_{l}=5.5 \mathrm{~m}^{2} \mathrm{~s}^{-1}$, taken from part III [8], were used in Eq. 58 .
Kinetic undercooling The kinetic undercooling for a pure metal with isotropic attachment kinetic at the interface is given by [4]

$\Delta T_{K}=\frac{R_{L}}{\mu_{k}}$

where $\mu_{k}$ is the attachment kinetics coefficient. For nickel, $\mu_{k} \approx 2 \times 10^{4} \mathrm{~ms}^{-1} \mathrm{~K}^{-1}$ [4]. The model in Eq. 60 with $\mu_{k} \approx$ $2 \times 10^{4} \mathrm{~ms}^{-1} \mathrm{~K}^{-1}$ was used to approximate $\Delta T_{K}$ for alloy 718, which is plotted in Fig. 2.

\section{References}

1. Draxler J, Edberg E, Andersson J, Lindgren L-E Modeling and simulation of weld solidification cracking, part I: a pore based crack criterion, to be published

2. Rappaz M, Drezet J-M, Gremaud M (1999) A new hot-tearing criterion. Metallurg Mater Trans A 30(2):449-455

3. Suyitno W, Katgerman L (2005) Hot tearing criteria evaluation for direct-chill casting of an Al-4.5 pct $\mathrm{Cu}$ alloy. Metall Mater Trans A 36(6):1537-1546

4. Dantzig JA, Rappaz M (2016) Solidification: -revised \& expanded. EPFL press

5. Coniglio N, Cross CE (2009) Mechanisms for solidification crack initiation and growth in aluminum welding. Metall Mater Trans A 40(11):2718-2728

6. Heinrich JC, Poirier DR (2004) Convection modeling in directional solidification. Comptes Rendus Mecanique 332(5-6):429-445

7. Grong O (1994) Metallurgical modelling of welding.pdf. Cambridge University Press, Cambridge

8. Draxler J, Edberg E, Andersson J, Lindgren L-E Modeling and simulation of weld solidification cracking, part III: simulation of cracking in varestraint tests of alloy 718 , to be published

9. Foster S, Carver K, Dinwiddie R, List F, Unocic K, Chaudhary A, Babu S (2018) Process-defect-structure-property correlations during laser powder bed fusion of alloy 718: role of in situ and ex situ characterizations. Metall Mater Trans A 49(11):57755798

10. Stefanescu DM (2015) Science and engineering of casting solidification. Springer

11. Kurz W, Giovanola B, Trivedi R (1986) Theory of microstructural development during rapid solidification. Acta Metallur 34(5):823830 
12. Rappaz M, David S, Vitek J, Boatner L (1990) Analysis of solidification microstructures in $\mathrm{Fe}-\mathrm{Ni}-\mathrm{Cr}$ single-crystal welds. Metallur Trans 21(6):1767-1782

13. Andersson J-O, Helander T, Höglund L, Shi P, Sundman B (2002) Thermo-calc \& dictra, computational tools for materials science. Calphad 26(2):273-312

14. Rappaz M, Jacot A, Boettinger WJ (2003) Last-stage solidification of alloys: theoretical model of dendrite-arm and grain coalescence. Metall Mater Trans A 34(3):467-479

15. Cengel YA (2010) Fluid mechanics. Tata McGraw-Hill Education
16. Sistaninia M, Phillion A, Drezet J-M, Rappaz M (2012) A 3D coupled hydromechanical granular model for simulating the constitutive behavior of metallic alloys during solidification. Acta Mater 60(19):6793-6803

17. Vernède $S$ (2007) A granular model of solidification as applied to hot tearing

Publisher's note Springer Nature remains neutral with regard to jurisdictional claims in published maps and institutional affiliations. 